\title{
Qualidade de vida, trabalho e apoio familiar de pessoas com fibromialgia
}

\author{
Family support and quality of life for people with fibromyalgia \\ Apoyo familiar y calidad de vida para personas con fibromialgia
}

Luana Maria Liborio da Mota ${ }^{1 *}$, Mariane Silva Queiroz ${ }^{1}$, Beatriz Tianeze de Castro ${ }^{1}$, Lorena Vieira Fernandez de Araújo ${ }^{1}$, Thais Oliveira Leite ${ }^{1}$, Bárbara Dielly Costa Balisa ${ }^{1}$, Shesllen Mikaelly Cruz Corrêa ${ }^{1}$, Juliano Gusmão de Oliveira', Fernanda Sales Alves Correia ${ }^{1}$, Rosângela Souza Lessa'.

\section{RESUMO}

Objetivo: Avaliar a qualidade de vida, o trabalho e o apoio familiar em pessoas com fibromialgia. Métodos: Trata-se de um estudo de corte transversal, com abrodagem quantitativa, com 102 fibromiálgicos. A coleta de dados ocorreu por meio de um questionário online composto por perguntas sobre perfil sociodemográfico e apoio familiar e o Questionário sobre o Impacto da Fibromialgia (QIF). Os dados quantitativos foram oriundos da ferramenta Google Forms e salvo em tabela do Microsoft Excel ®. O estudo foi aprovado por Comitê de Ética em Pesquisa. Resultados: Obteve-se dessa amostra um predomínio do sexo feminino 97,1\%, 63,7\% era pardo, $68,6 \%$ casado ou em união consensual/estável e 45,1\% com escolaridade até o ensino médio. Quanto às atividades remuneradas $65,7 \%$ responderam estar trabalhando. Na avaliação global do QIF atingiram uma média de 75,70 \pm 16,71 indicando elevado comprometimento da qualidade de vida. Conclusão: É preciso, então, atenção à saúde e ao bem-estar do fibromiálgico, compreensão familiar, empatia dos profissionais de saúde e novos estudos acerca da doença.

Palavras-chave: Fibromialgia, Qualidade de vida, Apoio familiar de paciente, Ambiente de trabalho.

\section{ABSTRACT}

Objective: To assess the quality of life, work and family support in people with fibromyalgia. Methods: This is a cross-sectional study, with a quantitative approach, with 102 patients with fibromyalgia. Data collection took place through an online questionnaire consisting of questions about sociodemographic profile and family support and the Questionnaire on the Impact of Fibromyalgia (QIF). Quantitative data came from the Google Forms tool and saved in a Microsoft Excel $\circledast$ table. The study was approved by the Research Ethics Committee. Results: From this sample, there was a predominance of $97.1 \%$ females, $63.7 \%$ were brown, $68.6 \%$ were married or in a stable/consensual union, and $45.1 \%$ had completed high school. As for paid activities, $65.7 \%$ said they were working. In the global assessment of the QIF, they reached a mean of $75.70 \pm 16.71$, indicating a high impairment of quality of life. Conclusion: It is necessary, then, attention to the health and well-being of fibromyalgia, family understanding, empathy of health professionals and further studies about the disease.

Key words: Fibromyalgia, Quality of life, Patient family support, Work environment.

\section{RESUMEN}

Objetivo: Evaluar la calidad de vida, el trabajo y el apoyo familiar en personas con fibromialgia. Métodos: Se trata de un estudio transversal, con abordaje cuantitativo, con 102 pacientes con fibromialgia. La recogida de datos se realizó a través de un cuestionario online compuesto por preguntas sobre perfil sociodemográfico y apoyo familiar y el Cuestionario sobre el Impacto de la Fibromialgia (QIF). Los datos cuantitativos provienen de la herramienta Google Forms y se guardan en una tabla de Microsoft Excel®. El estudio fue aprobado por el Comité de Ética en Investigación. Resultados: De esta muestra, hubo un predominio de $97.1 \%$ mujeres, $63.7 \%$ eran morenas, $68.6 \%$ estaban casadas o en unión estable / consensuada, y $45.1 \%$ habían terminado la escuela secundaria. En cuanto a las actividades remuneradas, el $65,7 \%$ dijo estar trabajando. En la valoración global del QIF alcanzaron una media de $75,70 \pm 16,71$, lo que indica un alto compromiso en la calidad de vida. Conclusión: Es necesario, entonces, la atención a la salud y el bienestar de la fibromialgia, la comprensión familiar, la empatía de los profesionales de la salud y más estudios sobre la enfermedad.

Palabras clave: Fibromialgia, Calidad de vida, Apoyo familiar del paciente, Ambiente laboral.

\footnotetext{
${ }^{1}$ Faculdade Santo Agostinho (FASA), Vitória da Conquista - BA. *E-mail: Luana_liboriomota@hotmail.com
} 


\section{INTRODUÇÃO}

A Fibromialgia (FM) é uma síndrome clínica caracterizada por dor e sensibilidade musculoesquelética crônica e generalizada, fadiga, sono não restaurador, disfunção cognitiva, depressão e ansiedade. A dor crônica insidiosamente reúne fatores desencadeantes e mantenedores, sendo estes de origem somática, psicológica e ambiental. Dessa forma, esse estado persistente de dor afeta a vida da pessoa com FM (SOUZA JBD e PERISSINOTTI DMN, 2018; CORREIA LC, et al., 2018; HEYMANN RE, et al., 2017; CABOMESEGUER A, et al., 2017).

A prevalência da FM pode variar de $0,2 \%$ até $5,4 \%$ a depender do continente, sendo esta mais comum no sexo feminino. Um inquérito telefônico realizado no Brasil, com 723 respondentes evidenciou que 1,79\% das pessoas relataram apresentar o diagnóstico de FM, numa proporção de 1:5,6 para homens e mulheres, respectivamente (MARQUES AP, et al., 2017; SOUZA JBD e PERISSINOTTI DMN, 2018).

Apesar de ser uma doença de causa desconhecida, os indivíduos com FM possuem alterações em alguns neurotransmissores, como substância $P$, noradrenalina e serotonina, que interferem na forma de percepção e perpetuação da dor. Desse modo, o diagnóstico da FM é essencialmente clínico, de acordo com análise dos sintomas característicos e sua correlação com o impacto na qualidade de vida do fibromiálgico (SOUZA JBD e PERISSINOTTI DMN, 2018; COSTA SML, et al., 2020).

Além disso, a complexidade do conceito de qualidade de vida quando a caracteriza como a percepção subjetiva individual sobre sua posição no contexto sociocultural e a relação disto com as suas expectativas e objetivos. Ademais, essa expressão engloba vários aspectos, como saúde, estado físico e mental, grau de independência e relações pessoais e com o ambiente (MELO GAD, et al., 2019).

Nessa perspectiva, é possível compreender a interferência das limitações funcionais da FM, principalmente quando associada aos níveis elevados de dor e aos aspectos psicológicos. O impacto negativo na qualidade de vida ocorre no contexto familiar, no ambiente de trabalho e dificulta a realização tarefas que exijam fisicamente do fibromiálgico, até quando se trata de atividades de lazer. Além da adaptação quanto a perda de saúde, a longa busca pelo diagnóstico, a falta de conhecimento e compressão das pessoas sobre a FM podem também contribuir negativamente no processo saúde-doença (MELO GAD, et al., 2019).

Outro aspecto de extrema importância para garantir o bem-estar da pessoa com FM é uma boa rede de apoio familiar, considerada definidora no enfrentamento da doença. $O$ apoio dos membros da família dar-seá no auxílio nas atividades domésticas, no incentivo a realização de atividade física, no acompanhamento nas consultas e orientações médicas e no suporte emocional durante as crises. Todavia, o sentimento de culpa e de invalidez acompanham o fibromiálgico que, muitas vezes, opta pelo isolamento a ter que sobrecarregar a família (MAZO JPS e ESTRADA MG, 2018). Assim, o presente estudo objetiva avaliar a qualidade de vida, 0 trabalho e 0 apoio familiar em pessoas com fibromialgia.

\section{MÉTODOS}

Trata-se de um estudo de corte transversal com abordagem quantitativa, caracterizado por analisar a causa e efeito num dado momento, mesmo que a causa já exista há algum tempo, isto é, seja somente uma particularidade do indivíduo (ESPINDOLA PL, et al., 2018; MAZO JPS e ESTRADA MG, 2018). A região de saúde do município que o estudo foi desenvolvido atende as demandas de saúde local e de mais 18 cidades circunvizinhas, totalizando 670.285 habitantes segundo o Plano Diretor de Regionalização da Bahia. Sendo assim, o munícipio é referência em serviços de média e alta complexidade que complementam a assistência à população das demais cidades (MARQUES AP, et al., 2017; BAHIA, 2018).

Foram adotados todos os padrões de aleatoriedade na seleção dos participantes. Os mesmos foram convidados a participar da pesquisa por meio da divulgação nas redes sociais e em consultórios reumatológicos utilizando vídeos com explicações acerca da pesquisa. A amostra foi do tipo probabilística e o cálculo para seleção da amostra seguiu os seguintes parâmetros: população de homens e mulheres, residente em um munícipio baiano de médio porte, segundo estimativa do Instituto Brasileiro de Geografia e 
Estatística (341.128 mil habitantes), prevalência estimada de fibromialgia no Brasil (20\%), erro tolerável amostral (5\%) e intervalo de confiança de $95 \%$.

A coleta de dados ocorreu online por meio do Google Forms, sendo o questionário enviado pelas redes sociais e/ou e-mail aos fibromiálgicos que se voluntariaram a participar da pesquisa. Os critérios de inclusão foram: indivíduos de ambos os sexos, maiores de 18 anos, atendidos pelos serviços de saúde no município estudado e que já possuíam o diagnóstico médico confirmado de fibromialgia. Dessa forma, obteve-se a participação de 102 respondentes.

A ferramenta gratuita Google Forms auxilia na produção e envio do questionário e tabulação dos dados quantitativos. No formulário constava o Termo de Consentimento Livre e Esclarecido (TCLE), os objetivos da pesquisa e orientações gerais da pesquisa, seguindo às exigências éticas da Resolução 466/2012. O estudo foi aprovado pelo Comitê de Ética em Pesquisa da Faculdade de Medicina de Itajubá (FMIT), sob o parecer 4.036.120, tendo a coleta iniciado somente após aprovação.

Ademais, o formulário foi composto por um bloco de perguntas para analisar o perfil sociodemográfico, como idade, cor da pele, sexo biológico, estado civil, escolaridade e dados ocupacionais. Neste último item, foi especificado o tipo de profissão, carga horária e os motivos da não realização dessa atividade, caso não se aplicasse a rotina do participante.

Para avaliar o impacto da fibromialgia na qualidade de vida utilizou-se o Questionário Sobre o Impacto da Fibromialgia (QIF) na versão traduzida para o português. O QIF é um questionário validado com 10 perguntas, sendo que os aspectos analisados foram: capacidade funcional, sentir-se bem, faltas no trabalho, interferência dos sintomas no trabalho, dor, fadiga, rigidez matinal, cansaço matinal, ansiedade e depressão (MARQUES AP, et al., 2006).

A primeira variável (capacidade funcional) é composta por 10 perguntas relacionadas com a funcionalidade física na vida diária, ou seja, fazer compras, lavar roupa, cozinhar, limpar a casa, arrumar a cama, andar vários quarteirões, visitar parentes ou amigos, cuidar do quintal, dirigir ou andar de ônibus. Cada uma deveria ser respondida numa escala do tipo Likert, sendo de zero (sempre) a três (nunca), totalizando pontuação máxima de 30 pontos. Na segunda e terceira variáveis do QIF utilizou-se uma escala de 0 a 7, sendo que a aquela é marcada inversamente para que o maior número (7) corresponda à insuficiência e essa é marcada diretamente. Já nos sete últimos itens do questionário foi usada uma escala visual analógica de 0 a 10 (AZEVEDO VF, et al., 2011; MARTINS R, et al., 2016).

O QIF foi avaliado adotando os seguintes parâmetros: primeira variável somada e dividida por três; os itens 2 e 3 multiplicados por 1,43 individualmente e os itens 4 a 10 foram somente somados, podendo o escore variar de 0 a 100 . Quanto maior a pontuação atingida no QIF, mais elevado foi o impacto da fibromialgia na qualidade de vida dos pesquisados (AZEVEDO VF, et al., 2011; MARQUES AP, et al., 2006).

Os dados quantitativos foram oriundos da ferramenta Google Forms e salvo em tabela do Microsoft Excel $\AA$, onde procedeu a limpeza e tabulação dos dados, em seguida realizou-se a análise utilizando o programa estatístico software R versão 3.0.3 (2014-03-06), Copyright (C) 2014 The R Foundation for Statistical Computing. O impacto da fibromialgia na qualidade de vida dos participantes do estudo foi descrito por meio de frequências simples e percentuais, média e desvio padrão, sendo expressos em tabelas e gráficos.

\section{RESULTADOS E DISCUSSÃO}

De acordo com o perfil sociodemográfico dos 102 FM participantes deste estudo, houve uma predominância de acometimento no sexo feminino $(97,1 \%)$ e na faixa etária de 41 a 50 anos de idade, com média de 45,03 $\pm 8,64$. A maior parte dos respondentes declarou ser pardo $(63,7 \%)$, casado ou em união consensual/estável $(68,6 \%)$ e com ensino médio (45,1\%) (Tabela 1). Estes dados corroboram com outros estudos, exceto em relação à etnia que apontam a raça branca como a mais comumente afetada pela FM (MARTINEZ JE, et al., 2017; MELO GAD, et al., 2019; OLIVEIRA JPR, et al., 2019). 
Tabela 1 - Perfil sociodemográfico dos fibromiálgicos em um munícipio baiano, Brasil, 2020 ( $n=102)$.

\begin{tabular}{lcc} 
& Variáveis & Frequência \\
\cline { 2 - 3 } & $\mathbf{N}$ & $\%$ \\
\hline Idade anos $\left(\mathrm{M}^{*}=45,03 \pm 8,64\right)$ & - & - \\
\hline Faixa etária (anos) & 4 & 3,9 \\
\hline $26-30$ & 22 & 21,6 \\
$31-40$ & 53 & 52 \\
$41-50$ & 19 & 18,6 \\
$51-60$ & 4 & 3,9 \\
$61-72$ & & \\
\hline Sexo & 99 & 97,1 \\
\hline Feminino & 3 & 2,9 \\
Masculino & & 19,6 \\
\hline Cor da pele & 20 & 63,7 \\
\hline Branca & 65 & 13,7 \\
Parda & 14 & 2,0 \\
Preta & 2 & 1,0 \\
Amarela & 1 & 16,7 \\
Indígena & & 68,6 \\
\hline Estado civil & 17 & 12,7 \\
\hline Solteira & 70 & 2 \\
Casada ou União consensual/estável & 13 & 11,8 \\
Divorciada & 2 & 45,1 \\
Viúva & & 28,4 \\
\hline Escolaridade & 12 & 14,7 \\
\hline Ensino Fundamental & 46 & \\
Ensino médio & 29 & \\
Ensino superior & 15 & \\
Pós-graduação & & \\
\hline
\end{tabular}

Legenda: $\mathrm{M}^{*}=$ média de idade. Fonte: Mota LMLD, et al., 2020.

Com relação à avaliação global do QIF, 73,5\% dos fibromiálgicos obtiveram um escore entre 70 e 100 pontos, indicando um alto índice de impacto da fibromialgia na qualidade de vida. A média do escore foi de $75,70 \pm 16,71$ no total de participantes, com um mínimo de 23,72 e máximo de 98,35 . Pesquisas realizadas por Lorena SB, et al. (2016) e Oliveira JPR, et al. (2019) demonstraram valor do escore do QIF de 75,9 e 65,5 respectivamente, semelhante ao encontrado nesse estudo, denotando baixa qualidade de vida.

Um estudo em Portugal (118 mulheres e 22 homens), utilizando o QIF, destacou o grau de incapacidade dos fibromiálgicos. Sendo assim, 50\% referiram a doença como bastante incapacitante, $45,7 \%$ a percebiam como moderadamente incapacitante e somente $2,9 \%$, sendo todas mulheres, declararam a FM como extremamente incapacitante. O escore total do QIF variou entre zero e 90, sendo possível notar o quão relevante é o impacto da FM, já que valores acima de 50 expressam má qualidade de vida em pessoas com FM (MARTINS R, et al., 2016).

No tocante a capacidade funcional foi possível destacar que a maioria dos respondentes do presente estudo só consegue realizar as atividades apontadas "de vez em quando", evidenciando o grau de limitação das atividades de vida diária. Apenas duas dimensões, frequência com que cozinha e arruma a cama, apresentaram respostas positivas, de modo que a resposta "sempre" foi assinalada por $45,1 \%$ e $39,2 \%$ dos participantes, respectivamente (Figura 1).

Estudo realizado com mulheres com FM apontou que 32\% delas tinham grandes limitações para realização de algumas atividades corriqueiras como: limpar a casa, andar vários quarteirões e cuidar do quintal ou jardim, bem como $24 \%$ para subir lance de escadas e $18 \%$ na realização de compras. Tal dado vai de encontro aos resultados desta pesquisa, já que os participantes relataram uma limitação menor quando apontaram realizar essas atividades "de vez em quando". Diferentemente, cozinhar e arrumar a cama, também são sempre exercidas na vida diária. Essa diminuição da capacidade física impacta diretamente na dinâmica da família, 
de modo que está se torna o principal sistema de apoio na realização das atividades domésticas (ALMEIDA FGJD, 2013; COSTA LPA, 2017).

Figura 1 - Capacidade funcional dos fibromiálgicos com base no Questionário Sobre o Impacto da Fibromialgia, em um munícipio baiano, Brasil, 2020 ( $n=102)$.

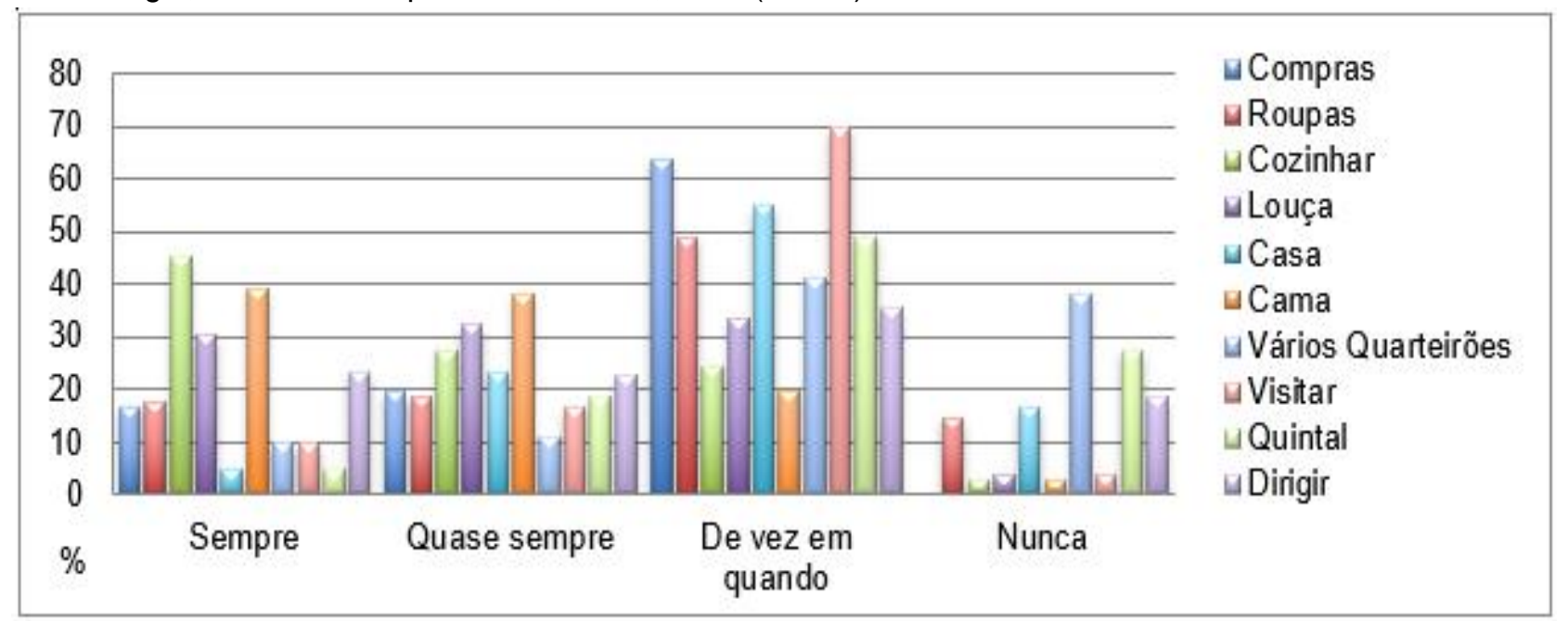

Fonte: Mota LMLD, et al., 2020.

Em relação às atividades remuneradas, $34,3 \%$ dos respondentes relataram não realizar nenhuma e, portanto, não possuir renda própria. Dentre os que responderam possuir um emprego $(65,7 \%)$, a maioria cumpria uma carga horária de trabalho semanal menor ou igual a 40 horas. A profissão que se destacou entre os fibromiálgicos pesquisados foi a de professor (10,8\%) (Tabela 2). Segundo a pesquisa de Collado A, et al. (2014) com 325 pacientes com FM da Atenção Primária, 45\% trabalhavam, porém apenas 34\% estavam ativos, pois, destes, $11 \%$ encontravam-se em licença médica devido à doença.

Tabela 2 - Características ocupacionais dos fibromiálgicos em um munícipio baiano, Brasil, 2020 (n=102).

\begin{tabular}{lcc}
\multicolumn{1}{c}{ Variáveis } & N & Frequência \\
\cline { 2 - 3 } & & $\%$ \\
\hline Atividade remunerada & 67 & 65,7 \\
\hline Sim & 35 & 34,3 \\
Não & 10 & 14,9 \\
\hline Profissão & 6 & 8,9 \\
\hline Professor & 6 & 8,9 \\
Servidor/Funcionário público & 5 & 7,5 \\
ACE $^{*}$ & & 7,5 \\
ACS & 5 & 6,0 \\
Técnico/Auxiliar administrativo & & 6,0 \\
Agente de limpeza & 4 & 40,3 \\
Empregada doméstica & 4 & \\
Serviços gerais & 27 & 70,1 \\
Técnico em enfermagem & & 9,9 \\
Autônomo & 47 & 9,0 \\
Outras & 14 & \\
\hline Carga horária de trabalho semanal & 6 & \\
\hline S40 horas & & \\
$>40$ horas & & \\
Não respondeu & & \\
\hline
\end{tabular}

Legenda: $\mathrm{ACE}^{*}=$ Agente de combate de endemias; $\mathrm{ACS}^{* *}=$ Agente comunitário de saúde.

Fonte: Mota LMLD, et al., 2020. 
A capacidade de trabalho diminuída do fibromiálgico pode impactar negativamente a qualidade de vida, haja vista que pessoas com FM quando comparadas com indivíduos com outras condições crônicas de dor, possuem um sofrimento mental mais evidente devido à percepção das suas limitações funcionais. Além disso, é importante ressaltar que essa dificuldade para realizar as atividades de vida diária e também as atividades laborais, exacerba ainda mais o quadro álgico, depressivo e ansioso, o que colabora para o círculo vicioso da doença (SOUZA JBD e PERISSINOTTI DMN, 2018; OLIVEIRA JPR, et al., 2019).

Dando destaque aos tipos de atividades laborais desenvolvidas pelos fibromiálgicos empregados, estes costumam trabalhar em empregos não manuais, incluindo ocupações de escritório, gerenciais ou associados a campos da saúde e educação. Já especificamente sobre a classe de professores, a mudança do setor educacional, que busca maior produtividade e eficiência desses profissionais, acaba por sobrecarregá-los. Muitos relatam elevado estresse profissional, desvalorização, baixa remuneração, carga horária exorbitante, quantidade excessiva de alunos, entre outros. Isso contribui para a somatização do sofrimento psicológico revertido em dor corporal e para o aumento do absenteísmo no trabalho (RIPPEL CB, et al., 2017; MATTOS RDS e LUZ MT., 2012; FITZCHARLES et al., 2017).

Em relação à jornada de trabalho, a literatura evidencia que a maior parte dos fibromiálgicos cumpre 31 a 40 horas por semana e que seus empregos demandam muito mentalmente. Nessa perspectiva, faz-se necessário à flexibilização das horas e ritmo da jornada de trabalho, na tentativa de possibilitar ao indivíduo com FM um ambiente de trabalho mais adequado a sua condição de saúde (RAKOVSKI C, et al., 2012; SILVA LCD, 2015).

No presente estudo, ao contrário do que foi encontrado na literatura, 30,4\% dos fibromiálgicos respondentes não haviam faltado nenhum dia ao trabalho nos últimos sete dias. Assumpção $A$, et al. (2010) comparou dois grupos com idade, sexo e índice de massa corporal (IMC) semelhantes diferenciando-se apenas pelo acometimento da FM. O grupo de fibromiálgicos relatou um maior número de faltas no trabalho do que o grupo controle. Essas faltas, porém não foram proporcionais ao grau elevado de comprometimento físico e mental encontrados.

Quanto a todas as demais dimensões do QIF (capacidade de trabalhar, intensidade da dor e do cansaço, disposição e rigidez matinal, ansiedade e depressão) os participantes relataram um grau elevado de influência da fibromialgia nesses aspectos. A maioria em todos os questionamentos respondeu na escala analógica 0 valor máximo (Figura 2). Martins R, el al. (2016) revela média maior que 7 para todas as variáveis supracitadas, correlacionado principalmente com maior intensidade da dor.

Figura 2 - Dimensões do Questionário Sobre o Impacto da Fibromialgia, em um munícipio baiano, Brasil, $2020(n=102)$.

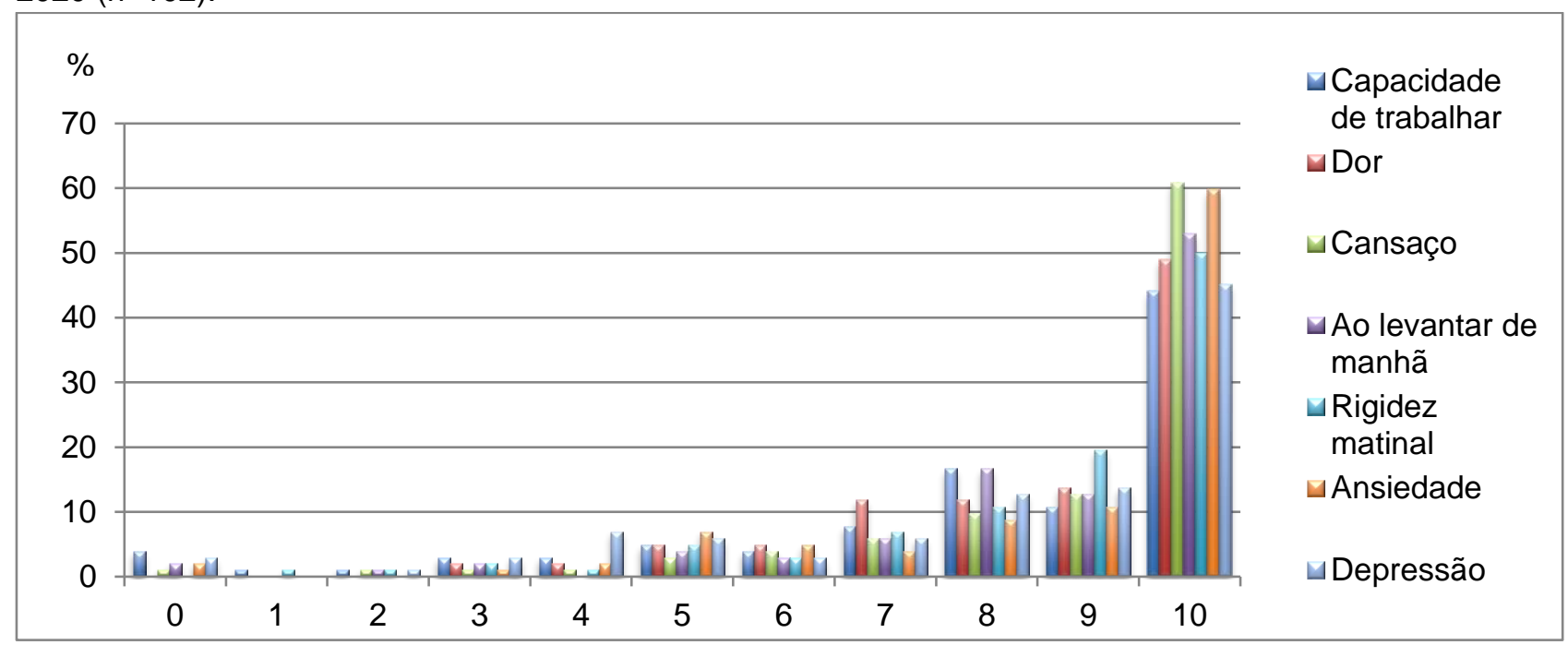

Fonte: Mota LMLD, et al., 2020. 
O paciente fibromiálgico vive uma realidade que cria sentimentos de vulnerabilidade e desamparo, que acarretam processos emocionais, incluindo a depressão. A variação de estados de humor (tensão, depressão, raiva, confusão, fadiga e baixo vigor) está associada à idade, ao fato de estar trabalhando ou não, a quantidade de sintomas e ao diagnóstico conclusivo de depressão. Como a prevalência da depressão em pessoas com fibromialgia é significativa e está correlacionada principalmente à dor e a pior qualidade de vida é frequente a sobreposição entre elas e, portanto, sejam confundidas (SOUZA BD e LAURENTI C, 2017; CONTE MS, et al., 2018).

Os fibromiálgicos possuem níveis mais elevados de substância $P$, neurotransmissor responsável pela perpetuação da percepção dolorosa nas fibras $C$ não mielinizadas, e níveis diminuídos de serotonina e noradrenalina, responsáveis por atuar nas vias inibitórias da dor. Isso denota anormalidades no processamento doloroso no sistema nervoso central (SNC) e, portanto, uma sensibilização persistente a dor. É esse intenso quadro álgico que interfere principalmente nas atividades de vida diária, sono, trabalho, aspectos emocionais, no autocuidado, vida social e sexual do indivíduo com FM (OLIVEIRA BR e CLARO RFT, 2020; COSTA SML, et al., 2020; SOUZA JBD e PERISSINOTTI DMN, 2018).

A compreensão da fibromialgia pelos familiares é instrumento importante para o enfrentamento da doença, dessa forma, quando questionados sobre isso $43,1 \%$ apontaram que a família compreende seu problema de saúde e somente $15,7 \%$ consideraram ruim o apoio familiar nas atividades domésticas e no cuidado dos seus aspectos físicos, emocionais e na sua saúde em geral. Contudo, 56,9\% acreditavam que os sintomas da fibromialgia afetam negativamente as relações familiares. Em relação ao apoio familiar é possível conjecturar que as famílias ainda encontram dificuldades em compreender e solidarizar-se quanto aos impactos e limitações que a fibromialgia traz para os indivíduos por ela acometidos. Uma pesquisa com 55 sócios da associação de fibromialgia do Principado de Asturias evidenciou que grande parte refere baixa satisfação familiar e reconhece um moderado impacto da doença (PÉREZ JMC e VÁZQUEZ IA, 2018).

Além disso, os familiares associam a FM à dor intensa e reconhecem que está atua produzindo mal-estar e limitações à vida dos fibromiálgicos, apesar de não possuírem um vasto conhecimento sobre a doença. Essa falta de informações sobre o real funcionamento da FM atua como fator limitante no seu controle. Além disso, é preciso ressaltar o papel do médico no processo de legitimidade da dor, de forma que o paciente e os familiares sejam devidamente orientados quanto ao curso crônico do quadro clínico e a dificuldade de manejo da doença, a qual necessita de um entendimento conjunto e apoio mútuo COSTA LPA, 2017).

O processo de compreensão com decorrer do tempo e a busca pelo entendimento da doença traz para a convivência familiar mudanças positivas, como compreender as limitações físicas e emocionais durante a realização das atividades habituais, participação do cônjuge no lar e na assistência durante o tratamento (MACEDO DCF, et al., 2015).

\section{CONCLUSÃO}

O presente estudo reforça a interferência negativa da FM na qualidade de vida do fibromiálgico devido às manifestações clínicas da doença é evidente, principalmente relacionado às limitações físicas e emocionais. A gravidade dos sintomas, especialmente da dor, fadiga e alterações humor, vão determinar o grau de sofrimento que recai sobre esse indivíduo e vai impactar diretamente nas relações familiares, no trabalho, nas atividades de vida diária, no bem-estar, na saúde mental e vida social e, consequentemente, traz impactos negativos na qualidade de vida. Por fim, é preciso estudos mais aprofundados sobre as questões relacionadas às atividades laborais e quais são as motivações para a saída do mercado de trabalho e também sobre a importância da participação familiar no processo saúde-doença do fibromiálgico.

\section{REFERÊNCIAS}

1. ALMEIDA FGJD. Impacto da fibromialgia na qualidade de vida de paciente assistidas no CRER, em Goiânia, Goiás. Dissertação (Mestrado em Ciências Ambientais e Saúde). Pós-graduação em Ciências Ambientais e Saúde. Universidade Católica de Goiás, Goiânia, 2013. 
2. ASSUMPÇÃO A, et al. Quality of life and discriminating power of two questionnaires in fibromyalgia patients: Fibromyalgia Impact Questionnaire and Medical Outcomes Study 36-item Short-Form Health Survey. Rev. Bras. Fisioter, 2010; 14(4): 284-9.

3. AZEVEDO VF, et al. FIQ score em pacientes fibromiálgicos com e sem espondilite anquilosante: análise comparativa. Rev. Med. Res., 2011; 13(3): 000-000.

4. BAHIA. Diretoria de Modernização Administrativa. Regiões de Saúde do Estado da Bahia. 2018. Disponível em: <http://www1.saude.ba.gov.br/mapa_bahia/result_REGIAO_SAUDEch.asp?REGIAO_SAUDE=Vit\%F3ria\%20da\%2 0Conquista>. Acesso em: 4 de setembro de 2020.

5. CORREIA LC, et al. Efeito do treinamento resistido na redução da dor no tratamento de mulheres com fibromialgia: revisão sistemática. Rev Bras Ciênc Mov., 2018;26(2):170-5.

6. CABO-MESEGUER A, et al. Fibromialgia: prevalência, perfiles epidemiológicos y costes económicos. Med. Clin., 2017; 149(10): 441-448.

7. COLLADO A, et al. Work, family and social environment in patients with Fibromyalgia in Spain: an epidemiological study: EPIFFAC study. BMC Health Serv Res., 2014; 14:513.

8. CONTE MS, et al. Fibromialgia: atividade física, depressão e qualidade de vida. Medicina (Ribeirão Preto, Online), 2018; 51(4): 281-90.

9. COSTA LPA. A família como rede de apoio às pessoas com fibromialgia: estratégias de cuidado interdisciplinar. Dissertação (Mestrado em Enfermagem, Saúde e Sociedade) - Programa de Pós-graduação em Enfermagem, Saúde e Sociedade. Universidade do Estado do Rio de Janeiro, Rio de Janeiro, 2017.

10. COSTA SML, et al. Aspectos clínicos e principais formas de tratamento para Fibromialgia-Revisão de Literatura. Research, Society and Development, 2020; 9(11): e729119495.

11. ESPINDOLA PL, et al. Data governance applied to information science: analysis of a scientific data system for the health area/governanca de dados aplicada a ciencia da informacao: analise de um sistema de dados cientificos para a area da saude/gobernanza de datos aplicada a la ciencia de la informacion: analisis de un sistema de datos cientificos para el area de la salud. Revista digital de biblioteconomica e ciencia da informação, 2018; 16(3): 274-299.

12. FITZCHARLES et al. The Association of Socioeconomic Status and Symptom Severity in Persons with Fibromyalgia. The Journal of Rheumatology, 2017; 24(7): 1398-404.

13. HEYMANN RE, et al. Novas diretrizes para o diagnóstico da fibromialgia. Ver. Bras. de Reumato., $2017 ; 57(2)$ : 467 476.

14. LORENA SB, et al. Evaluation of pain and quality of life of fibromyalgia patients. Rev. Dor., 2016; 17(1): 8-11.

15. MACEDO DCF, et al. Representações sociais de conjugalidade e fibromialgia: Desdobramentos na dinâmica conjugal. Temas em Psicologia, 2015; 23(4): 987-1002.

16. MARQUES AP, et al. A prevalência de fibromialgia: atualização da revisão da literatura. Ver. Bras. de Reumato., 2017; 57: 356-363.

17. MARQUES AP, et al. Validação da versão brasileira do Fibromyalgia Impact Questionnaire (FIQ). Rev. Bras. Reumatol., 2006; 46(1): 24-31.

18. MARTINEZ JE, et al. EpiFibro (Registro Brasileiro de Fibromialgia): dados sobre a classificação do ACR e preenchimento dos critérios diagnósticos preliminares e avaliação de seguimento. Ver. Bras. de Reumatol., 2017; 57(2): 129-133.

19. MARTINS R, et al. Fibromyalgia impact on quality of life of patients. Revista Servir. 2016; 59: 44-49.

20. MATTOS RDS, LUZ MT. Quando a perda de sentidos no mundo do trabalho implica dor e sofrimento: um estudo de caso sobre fibromialgia. Physis Revista de Saúde Coletiva, 2012; 22(4): 1459-1484.

21. MAZO JPS, ESTRADA MG. Implications of chronic pain on the quality of life of women with fibromyalgia. Psicol. Estud., 2018; 23: e38447.

22. MELO GAD, et al. Análise dos perfis sociodemográfico e clínico de mulheres com fibromialgia. Temas em saúde, $2019 ; 19(5)$.

23. OLIVEIRA JPR, et al. O cotidiano de mulheres com fibromialgia e o desafio interdisciplinar de empoderamento para o autocuidado. Revista Gaúcha de Enfermagem, 2019; 40:e20180411.

24. OLIVEIRA BR, CLARO RFT. O papel do exercício físico em pacientes com fibromialgia. Revista MotriSaúde, 2020; 2(1).

25. PÉREZ JMC, VÁZQUEZ IA. Relación del apoyo social y familiar com la aceptación del dolor em pacientes con fibromialgia. International Journal of Psychology and Psychological Therapy, 2018; 18(2): 151-161.

26. RAKOVSKI C, et al. Association of employment and working conditions with physical and mental health symptoms for people with fibromyalgia. Disability \& Rehabilitation, 2012; 34(15): 1277-1283.

27. RIPPEL CB, et al. Paradigmas do trabalho docente: percepção de professores municipais afastados ou readaptados por estresse sobre os fatores causais. Inova Saúde, 2017; 6(1): 208-225.

28. SILVA LCD. Adequação do trabalho para trabalhadores portadores de fibromialgia. Caderno de saúde e desenvolvimento, 2015; 7(4): 73-80.

29. SOUZA JBD, PERISSINOTTI DMN. A prevalência da fibromialgia no Brasil: estudo de base populacional com dados secundários da pesquisa de prevalência de dor crônica brasileira. BrJP, 2018; 1(4): 345-348.

30. SOUZA BD, LAURENTI C. Uma interpretação molar da dor crônica na fibromialgia. Psicologia: Ciência e Profissão, 2017; 37: 363-377. 